\title{
THE MULTI-BAND EMISSION PROFILE IN GRB
}

\author{
X.-H. Zhao ${ }^{1,2}$ and J.-M. Bai ${ }^{1,2}$
}

\begin{abstract}
Gamma-ray burst (GRB) light curves generally are energy dependent, i.e., the higher the photon energy, the narrower the pulse. In addition, the peak of the pulse at lower energies is usually delayed compared with that in higher energy. The phenomenon even exists in some X-ray flares and following optical flares. However, its origin is still unclear. Here we calculate the emission in different bands produced from different-thickness shocked shell due to radiative cooling, with the equal arrival time volume effect considered. We find the emission profile is energy dependent and that the light curves show spectral lag, which are consistent with the observations. This can be an explanation of the energy dependence of light curve and spectral lag in GRB, X-ray flare and following optical flare.
\end{abstract}

\section{Introduction}

Gamma-ray burst (GRB) light curves generally were found to be energy dependent in the BATSE/CGRO era, i.e., the higher the photon energy, the narrower the pulse with a power-law slope of $\sim-0.4$ (Fenimore et al. 1995; Norris et al. 1996). And the pulse at lower energy is usually lagged compared with that in higher energy (Norris et al. 2000). There also exists spectral lag in some X-ray flares and simultaneous optical observation (e.g., Kruhler et al. 2009; Rossi et al. 2011). Some explanations for the two phenomena have been proposed, including the radiative cooling and curvature effect. However, they both confront some severe problems in explaining the observations. The radiative cooling requires too low magnetic field, inconsistent with the current models (e.g., Sari \& Piran 1997; Piran 1999). The spectrum lag due to curvature effect is too small to fit the observation (e.g., Shen et al. 2005). Here we considered the synchrotron cooling

\footnotetext{
${ }^{1}$ Key Laboratory for the Structure and Evolution of Celestial Objects, Chinese Academy of Sciences, Kunming 650011, China

2 National Astronomical Observatories/Yunnan Observatory, Chinese Academy of Sciences, PO Box 110, Kunming 650011, China
} 
will lead to different survival time for different energy of electrons behind internal shock so that the emission in different bands comes from shells with different width. Our motivation is explaining the two observations. We calculated the light curves with the equal arrival time volume effect considered in the second section. Discussion and conclusions are presented in the third section.

\section{Shock hydrodynamics and light curves}

GRB is usually considered to come from internal shocks, i.e., the GRB central engine launches a lot of shells with different speeds and shocks will be produced when fast shells catch up with slow shells and collisions happen. The shocked electrons will give rise to the observed GRB by synchrotron or inverse Compton radiation. Suppose that a fast shell with speed of $\beta_{r}$ catches up with a slow shell with speed of $\beta_{s}$ and the two shells will undergo a full inelastic collision, where the width of both shells in the observed frame is $\Delta=R / \gamma_{s}^{2}, R=2 \gamma_{s}^{2} c \delta t$ and $\gamma_{s}$ being the radius of internal shock ( $\delta t$ is the variability time scale) and the Lorentz factor of slow shell, respectively. Two shocks, a reverse shock and a forward shock, will develop. For simplicity, here we only consider the reverse shock. The electrons after the shock will be accelerated. With the shock propagating in the fast shell, a "hot" region will form, in which the electrons have not yet had enough time to cool (safely neglecting the accelerating time of electrons). The width of the hot region is

$$
\Delta_{h}=\gamma_{m} t_{c o o l}^{\prime} c\left(\beta_{m}-\beta_{r s}\right),
$$

where $t_{\text {cool }}^{\prime}$ is the comoving cooling Lorentz factor. For synchrotron emission, $t_{c o o l}^{\prime}$ is given by

$$
\nu^{\prime}=\frac{q_{e} B}{2 \pi m_{e} c} \gamma_{e}^{2}, \quad P_{s}^{\prime}=\frac{4}{3} \sigma_{T} c \frac{B^{2}}{8 \pi} \gamma_{e}^{2}, \quad t_{\text {cool }}^{\prime}=\frac{3}{\sigma_{T}}\left(\frac{2 \pi m_{e} c q_{e} D}{B^{3} \nu}\right)^{1 / 2} .
$$

$\gamma_{m}$ is the Lorentz factor of merged shell, which can be given $\gamma_{m}=\sqrt{\frac{\gamma_{f} m_{f}+\gamma_{s} m_{s}}{m_{f} / \gamma_{f}+m_{s} / \gamma_{s}}}$, where $\gamma_{f}(s)$ is the Lorentz factor of fast(slow) shells. $B$ is the magnetic field behind the shock (in the comoving frame), given by $B=\sqrt{\frac{2 L_{i s o} \epsilon_{B}}{c R^{2} \gamma_{m}^{2} \epsilon_{e}}}$, where $L_{i s o}$ is the observed isotropic luminosity, $\epsilon_{e}$ and $\epsilon_{B}$ are the fractions of internal energy distributed to electrons and magnetic field. $D=1 /[\gamma(1-\beta \mu)]$ is the Doppler factor. For an infinity thin shell and for a given time, the observed emission is from a surface symmetric around the line of sight, the equal arrival time surface, which is described by $R=\frac{\beta c T}{1-\beta \mu}$. That means the emission is from an area. If the shell is not thin, for an observed time, $T$, the emission is from an equal time volume. The flux density is given by (Granot et al. 1999)

$$
F_{\nu}(T)=2 \pi \frac{1+z}{d_{L}^{2}} \int_{\mu_{0}}^{1} d \mu \int_{R_{i n}(\mu, T)}^{R_{e x}(\mu, T)} r^{2} d r D^{2} j_{\nu^{\prime}}^{\prime}\left(\Omega^{\prime}, \nu \gamma(1-\beta \mu), r, t\right)
$$



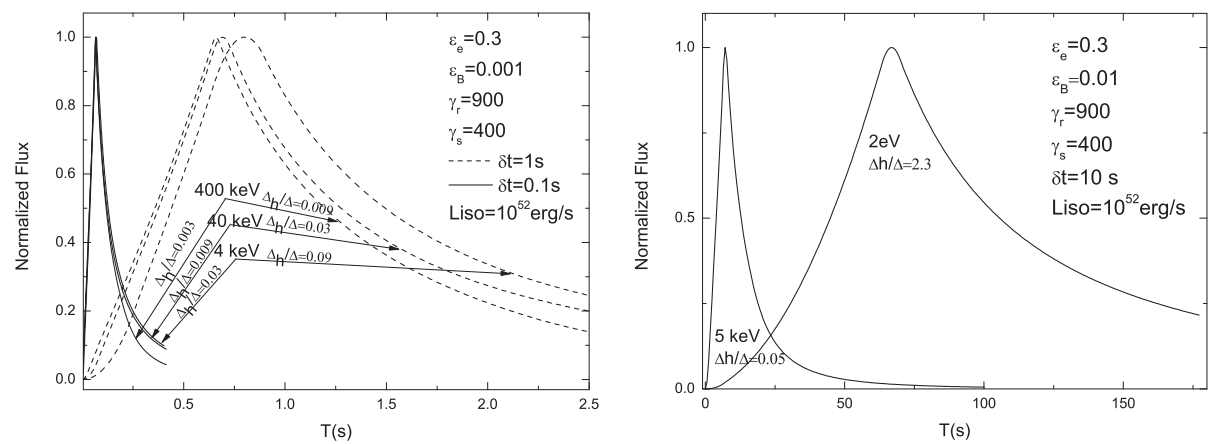

Fig. 1. The calculated Light curves in different bands.

$R_{\text {in }}$ and $R_{\text {ex }}$ are defined in Zhao et al. (2012), describing the emission is from an equal time volume. The comoving emissivity is given by $j_{\nu^{\prime}}^{\prime}\left(\Omega^{\prime}, \nu^{\prime}, r, t^{\prime}\right)=$ $\frac{d\left[n_{e}^{\prime}\left(r, t^{\prime}\right) P^{\prime}\left(\nu^{\prime}\right)\right]}{d \Omega d \nu^{\prime}}=\frac{n_{e}^{\prime}\left(r, t^{\prime}\right) P_{\nu^{\prime}}^{\prime}\left(\nu^{\prime}\right)}{4 \pi}$. Here $n_{e}^{\prime}$ is the comoving electron number density, which is the function of $r$. The electron distribution is power law form with the slope of $p=2.1$, and the corresponding synchrotron spectrum is broken power law with the low energy slope of $1 / 3$ and the high energy slope of $-(p-1) / 2$. The calculated light curves are shown in Figure 1. The spectrum lag and the pulse width extending in lower energy can be found. The spectrum lag is caused by a combination effect of shell geometry and synchrotron cooling. The softer the photon and thus the longer the cooling time of electrons, the wider the hot shell $\left(\Delta_{h}\right)$. The peak of a pulse for a wider hot shell will appears later, which will produce a lag. And a wider hot shell will also produce a wider pulse due to the equal arrival time volume effect.

\section{Discuss and conclusions}

The shocked shells with different thickness in different bands and equal arrival time volume may explain the observed energy dependence of light curve and spectral lag in GRB, X-ray flare and following optical flare.

\section{References}

Fenimore, E., in't Zand, J., Norris, J., Bonnell, J., \& Nemiroff, R., 1995, ApJ, 448, L101

Kruhler, T., Greiner, J., McBreen, S., et al., 2009, ApJ, 697, 758

Granot, J., Piran, T., \& Sari, R., 1999, ApJ, 513, 679

Norris, J.P., Nemiroff, R.J., Bonnell, J.T., et al., 1996, ApJ, 459, 393

Norris, J.P., Marani, G.F., \& Bonnell, J.T., 2000, ApJ, 534, 248

Rossi, A., Schulze, S., Klose, S., et al., 2011, A\&A, 529, 142

Sari, R., \& Piran, T., 1997, MNRAS, 287, 110

Shen, R.-F., Song, L.-M. \& Li, Z., 2005, ApJ, 362, 59

Zhao, X.-H., \& Bai, J.-M., 2012, in preparation 
